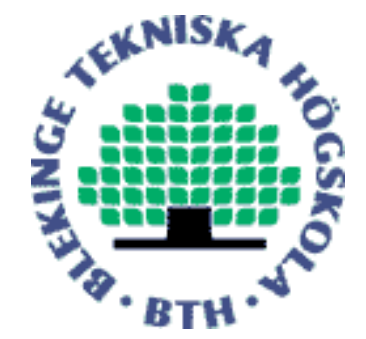

Copyright (C) 2014 IEEE.

Citation for the published paper:

On Power Consumption of Wireless Sensor Nodes with Min(N,T) Policy in Spectrum Sharing Systems

Charles Kabiri, Hans-Jürgen Zepernick, Tran Hung

IEEE Vehicular Technology Conference

2014 Seoul, Korea

This material is posted here with permission of the IEEE. Such permission of the IEEE does not in any way imply IEEE endorsement of any of BTH's products or services Internal or personal use of this material is permitted. However, permission to reprint/republish this material for advertising or promotional purposes or for creating new collective works for resale or redistribution must be obtained from the IEEE by sending a blank email message to pubs-permissions@iee.org.

By choosing to view this document, you agree to all provisions of the copyright laws protecting it. 


\title{
On Power Consumption of Wireless Sensor Nodes with Min(N,T) Policy in Spectrum Sharing Systems
}

\author{
Charles Kabiri ${ }^{1}$, Hans-Jürgen Zepernick ${ }^{1}$, and Hung Tran ${ }^{2}$ \\ ${ }^{1}$ Blekinge Institute of Technology \\ SE-371 79 Karlskrona, Sweden \\ E-mail: \{chk, hjz\}@bth.se \\ ${ }^{2}$ National Institute of Education Management, Information Technology Department \\ 31 Phan Dinh Giot Street, Thanh Xuan District, Hanoi, Vietnam \\ E-mail: tranhungemail@gmail.com
}

\begin{abstract}
In this paper, we analyze the power consumption of wireless sensor nodes with $\min (N, T)$ policy and M/G/1 queue in the presence of Nakagami- $m$ fading. In particular, this system setting is applied to a wireless sensor node operating in a cognitive radio system as secondary user in the presence of a primary user. As such, not only the queue policy influences the power consumption but also the interference power constraint imposed on the wireless sensor node by the primary user. Thus, a queued sleep/wake-up strategy is analyzed in order to mitigate the average power consumption of a sensor node using $\min (N, T)$ policy in the context of an M/G/1 queue and a spectrum sharing environment in the presence of signal fading. Numerical examples are presented to illustrate the impact of queuing parameters and fading channel on the power consumption of a wireless sensor node.
\end{abstract}

\section{INTRODUCTION}

New applications in the area of wireless sensor networks such as healthcare, environmental monitoring, disaster management, target surveillance and industrial control, are characterized by high data rates and low latency. Some of these applications will require communication over shared spectrum. This is due to the high density of deployed wireless sensor networks (WSNs) and the fact that it is often not economical to allocate a dedicated spectrum band to WSNs. However, sharing a spectrum band with a primary (licensed) system compromises the reliability and performance of both systems due to interference among the systems. As such, limited bandwidth, computing capacity, data delivery delay, severe energy constraints and severe fading conditions can make the design of WSNs more challenging [1].

Apparently, the operational lifetime of sensor nodes is an important issue in WSNs. As the lifetime of a sensor node is related to its energy consumption, sleep/wake-up schemes and energy efficient routing protocols are two prominent techniques used in WSNs to enhance lifetime [1]. In [2], sleep/wake-up strategies in cognitive radio-based wireless sensor networks are evoked. As most of the energy in sensor nodes is consumed during packet transmission, queuing theory can be an attractive tool to analyze and design efficient power allocation schemes. Furthermore, queuing performance measures in spectrum sharing systems have attracted considerable attention in recent work. In spectrum sharing scenarios, the authors of
[3] investigated the queueing behavior of a secondary user (SU) communicating over the channel licensed to the primary user (PU).

In queued sleep/wake-up schemes, to wake up a node from an idle state to the busy state means turning on the radio server and is dealt with by duty cycle schemes [4]. In the context of medium access control (MAC) protocols, the respective sleep/wake-up strategy plays a key role in the level of power savings in sensor nodes. Some MAC protocols such as IEEE 802.11, sensor MAC (S-MAC), sparse topology and energy management (STEM), or Berkeley (B-MAC) use the concept of queued wake-up where a threshold is used to control the average time of turning on the data radio and the latency for the buffered data packets. In this regards, the sensor node triggers the data radio only when the queue holds $N$ packets. The queued packets are transmitted thereafter, in a burst, as soon as the access to the transmission medium is established. It is an interesting question to find the optimal value of $N$ in order to minimize the power consumption. This can be attained from the so called $N$-policy [5].

The results reported in [4] provide a feasible power-saving technique using the $N$ policy along with an $\mathrm{M} / \mathrm{M} / 1$ queue, i.e. a single sever where arrivals follow a Poisson process and service time is exponentially distributed. A threshold, $N$, is specified and used to control the average time of turning on the radio transmitter for the buffered data packets. In other words, when the queue holds $N$ packets, the sensor triggers the radio transmitter to start the transmission for the queued packets in a burst. Although the $N$-policy provides energy savings, a long waiting time may be caused as packets are buffered until the threshold is reached. To prevent such delays, the $T$-policy has been proposed to deal with the case that the number of queued packets does not reach the threshold $N$. The $T$-policy consists of triggering the radio as soon as the waiting time after the last busy period has reached a given threshold $T$. A combination of these two policies can be formulated as $\min (N, T)$ operation [6], [7].

Other works related to energy-efficiency in sensor networks are presented in [8], [9] and the references therein. In [9], a discrete-time Markov model is used that allows to study the trade-off between energy consumption and transfer delay in 
clustered WSNs. Queue aware threshold control policies for the scenarios with infinite and finite buffer have been proposed in [10].

In this paper, to analyze system performance, we consider a spectrum sharing system utilizing an M/G/1 queueing model, i.e. single server with Poisson arrival, service time following a general distribution, and $\min (N, T)$ policy. The contributions of this paper are as follows: 1) We derive an analytical model of a sensor node that uses the $\min (N, T)$ policy and operates in an underlay spectrum sharing environment. 2) Modeling the sensor node as an M/G/1 queue, we assess the power consumption in the idle and busy state. 3) Different to [11], where an error free wireless channel is assumed, we include the effect of channel fading into the analysis.

The rest of the paper is organized as follows. Section II describes the system model along with the queuing policy. Section III provides the performance analysis by deriving the first and second moment of service time of data packets and a queuing model with $\min (N, T)$ policy used for the power consumption function. In Section IV, numerical results of the power consumption function are presented. Finally, Section V concludes the paper.

\section{SySTEM MODEL}

Let us consider a spectrum sharing scenario as shown in Fig. 1 where a WSN operates in the presence of the primary receiver (PU-Rx) of the primary network. The secondary network comprises of wireless sensor nodes, i.e. a secondary transmitter (SU-Tx) and a secondary receiver (SU-Rx). The data packets arriving and queued in the SU-Tx, which acts as single server, are served in first-in first-out (FIFO) order. We assume that the arrival rate $\lambda$ at the SU-Tx follows a Poisson process and that service time has a general distribution. In order to cater for a wide range of fading conditions, we assume that packet transmission is subject to Nakagami- $m$ fading where $m$ denotes the fading severity parameter.

\section{A. Peak interference power constraint}

In underlay cognitive radio networks, the secondary transmitter must adjust its power to avoid harmful interference to the primary receiver. Then, the packet transmission time between SU-Tx and SU-Rx in the secondary network can be determined by

$$
T_{L}=\frac{1}{b \log _{2}\left(1+\gamma_{s}\right)}
$$

where $b=B / L, B$ is the system bandwidth, $L$ is the number of bits per packet, and $\gamma_{s}$ is the signal-to-noise ratio (SNR) at the SU-Rx. Given the maximum allowed interference power at the PU-Rx as $Q$, then the SNR in (1) can be expressed as

$$
\gamma_{s}=\frac{g_{1} Q}{g_{0} N_{0}}
$$

$g_{0}$ and $g_{1}$ are the channel power gains of the SU-Tx $\rightarrow$ PU-Rx and SU-Tx $\rightarrow$ SU-Rx links, respectively, following the Gamma distribution with unit mean. Further, $N_{0}$ denotes the variance of the additive white Gaussian noise at the SU-Rx.

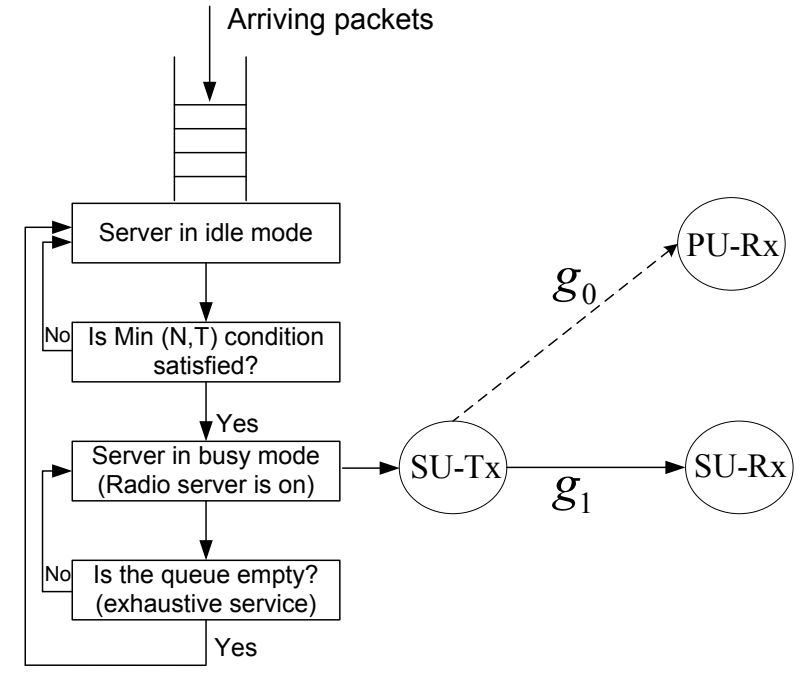

Fig. 1. System model of a wireless sensor network with an M/G/1 queue and $\min (N, T)$ policy at the transmitting sensor node that operates in a spectrum sharing environment with a primary receiver.

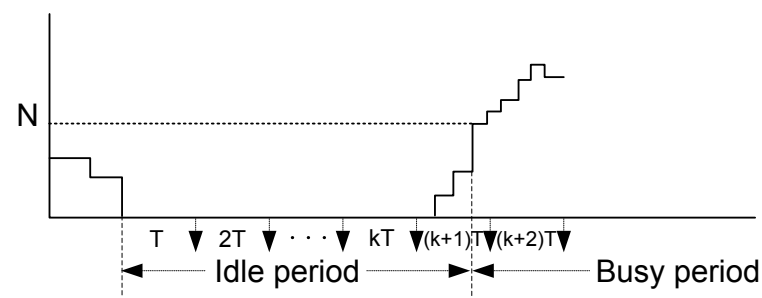

(a)

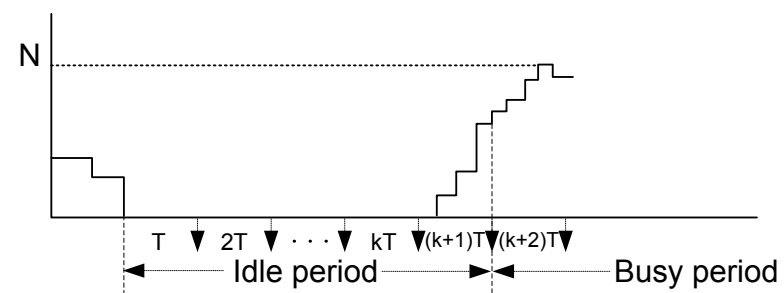

(b)

Fig. 2. Scenarios of transmitting service started by: (a) $N$-policy condition, (b) $T$-policy condition.

\section{B. Delay constraint}

For simplicity and to reduce further scheduling, we consider a simple stop-and-wait automatic repeat request protocol in the secondary network. As such, if a packet has been successfully received by the SU-Rx, an acknowledgement (ACK) is sent to the SU-Tx. Otherwise, a negative ACK (NACK) is sent which indicates a retransmission request to the SU-Tx. Here, we assume that the ACK/NACK are transmitted over an errorfree feedback channel with negligible delay. In order to limit the number of retransmissions, a packet is considered as being timed out and hence being dropped, if it has not been successfully received within a given period or service time $T_{L}$. As such, a packet is considered as being received successfully, 
if the following delay constraint is fulfilled:

$$
T_{L}<t_{\text {out }}
$$

\section{C. $\min (N, T)$ policy}

In this work, we allow the sensor node to be in idle state (monitoring) or busy state (transmitting) as illustrated in Fig. 2 [12]. In the idle state, the radio transmitter functionality is switched off to conserve energy while it is switched on in the busy state. In general, the $N$ and $T$ policy switches off the radio transmitter of the server (idle) when accumulating $N$ data packets in the queue and until reaching $T$ time units. Once $N$ packets are in the queue or the time $T$ is reached, the radio transmitter of the server is switched on (busy) and the packets in the queue are transmitted in a burst [7] [12].

\section{PERformance AnAlysis}

\section{A. First and second moment of service time $T_{L}$}

In order to derive the moments of $T_{L}$, we first need to obtain the cumulative distribution function (CDF) of the SNR $\gamma_{s}$ at the SU-Rx which can be formulated as

$$
F_{\gamma_{s}}(\gamma)=\operatorname{Pr}\left\{\frac{g_{1} Q}{g_{0} N_{0}}<\gamma\right\}
$$

According to [13], the CDF of $\gamma_{s}$ can be derived as

$$
\begin{aligned}
F_{\gamma_{s}}(\gamma) & =\left(\frac{m_{1}}{m_{0}}\right)^{m_{1}}\left(\frac{N_{0}}{Q}\right)^{m_{1}} \frac{\Gamma\left(m_{1}\right) \gamma^{m_{1}}}{\mathcal{B}\left(m_{0}, m_{1}\right)} \\
& \times{ }_{2} F_{1}\left(m_{1}, m_{0}+m_{1} ; 1+m_{1} ;-\gamma \frac{m_{1} N_{0}}{m_{0} Q}\right)
\end{aligned}
$$

where $m_{0}$ and $m_{1}$ are the fading severity parameters of links SU-Tx $\rightarrow$ PU-Rx and SU-Tx $\rightarrow$ SU-Rx, respectively. Furthermore, $\Gamma(\cdot)$ is the gamma function defined as $\Gamma(x)=$ $\int_{0}^{\infty} t^{x-1} e^{-t} d t$ [14, eq. (8.310.1)], ${ }_{2} F_{1}(., . ; . ;$.$) stands for the$ hypergeometric function [14, eq. (3.194.5)] and $\mathcal{B}(a, b)$ is the beta function [14, eq.(8.384.1)] defined as

$$
\mathcal{B}(a, b)=\frac{\Gamma(a) \Gamma(b)}{\Gamma(a+b)}
$$

On the other hand, the CDF of $T_{L}$ can be written as

$$
F_{T_{L}}(t)=\operatorname{Pr}\left(T_{L}<t\right)=1-\operatorname{Pr}\left(T_{L} \geqslant t\right)
$$

Substituting (1) into (7) along with some algebraic manipulation, the $\mathrm{CDF}$ of $T_{L}$ can be rewritten as

$$
F_{T_{L}}(t)=1-F_{\gamma_{s}}\left(2^{\frac{1}{b t}}-1\right)
$$

where $F_{\gamma_{s}}(\gamma)$ is the CDF determined in (5). Then differentiating (8) with respect to $t$, the probability density function (PDF) of $T_{L}$ can be determined as

$$
\begin{aligned}
f_{T_{L}}(t) & =\frac{d F_{T_{L}}(t)}{d t} \\
& =1 /\left[b m_{0} t^{2} \mathcal{B}\left(m_{0}, m_{1}\right)\right] \\
& \times 2^{\frac{1}{b t}} m_{1}\left[\frac{\left(2^{\frac{1}{b t}}-1\right) m_{1}}{m_{0}}\right]^{-1+m_{1}} \ln 2 \\
& \times\left(\frac{N_{0}}{Q}\right)^{m_{1}}\left[\frac{\left(2^{\frac{1}{b t}}-1\right) m_{1} N_{0}+m_{0} Q}{m_{0} Q}\right]^{-m_{0}-m_{1}}
\end{aligned}
$$

Let us recall that timeout occurs when packet transmission time is greater or equal to a given threshold $t_{\text {out }}$. In this case, a packet is dropped and the related timeout probability is formulated as

$$
\begin{array}{r}
P_{\text {out }}=\operatorname{Pr}\left(T_{L} \geq t_{\text {out }}\right)=1-F_{T_{L}}\left(t_{\text {out }}\right) \\
=F_{\gamma_{s}}\left(2^{\frac{1}{b_{\text {out }}}}-1\right)
\end{array}
$$

On the other hand, the PDF representing the case of packets not being timed out can be written as [15]

$$
f_{T_{L, s u c}}(t)= \begin{cases}\frac{f_{T_{L}}(t)}{1-P_{\text {out }}} & \text { for } 0 \leq t<t_{\text {out }} \\ 0 & \text { for } t \geq t_{\text {out }}\end{cases}
$$

Therefore, the first and second moment of the transmission time for packets that are not dropped, i.e., $T_{L, \text { suc }}=$ $\left\{T_{L} \mid T_{L}<t_{\text {out }}\right\}$ can be written as

$$
\begin{aligned}
E\left\{T_{L, \text { suc }}\right\} & =\int_{0}^{t_{\text {out }}} t f_{T_{L, \text { suc }}}(t) d t \\
& =\frac{1}{1-P_{\text {out }}} \int_{0}^{t_{\text {out }}} t f_{T_{L}}(t) d t \\
E\left\{T_{L, \text { suc }}^{2}\right\} & =\int_{0}^{t_{\text {out }}} t^{2} f_{T_{L, \text { suc }}}(t) d t \\
& =\frac{1}{1-P_{\text {out }}} \int_{0}^{t_{\text {out }}} t^{2} f_{T_{L}}(t) d t
\end{aligned}
$$

After performing some derivations, it can be shown that the first moment of $T_{L, \text { suc }}$ is given by

$$
E\left\{T_{L, \text { suc }}\right\}=\left(\frac{N_{0} m_{1}}{Q}\right)^{m_{1}} \frac{m_{0}^{m_{0}} Q^{m_{0}+m_{1}}}{\left(1-P_{\text {out }}\right) \mathcal{B}\left(m_{0}, m_{1}\right)} \Phi_{1}
$$

where $\Phi_{1}$ is defined as

$$
\Phi_{1}=\int_{\alpha}^{\infty} \frac{(u-1)^{m_{1}-1} u \ln u}{\left[m_{0} Q+m_{1} N_{0}(u-1)\right]^{m_{0}+m_{1}}} d u
$$

and $\alpha=2^{1 / b t_{\text {out }}}$. Similarly, the second moment of $T_{L, \text { suc }}$ can be obtained as

$$
E\left\{T_{L, \text { suc }}^{2}\right\}=\left(\frac{N_{0} m_{1}}{Q}\right)^{m_{1}} \frac{m_{0}^{m_{0}} Q^{m_{0}+m_{1}} \ln 2}{b\left(1-P_{\text {out }}\right) \mathcal{B}\left(m_{0}, m_{1}\right)} \Phi_{2}
$$

where $\Phi_{2}$ is given by 


$$
\Phi_{2}=\int_{\alpha}^{\infty} \frac{(u-1)^{m_{1}-1} u}{\left[m_{0} Q+m_{1} N_{0}(u-1)\right]^{m_{0}+m_{1}}} d u
$$

Let us return to service time $T_{L}$, which is the time needed by the server to process one packet. Without considering timeout, the mean service time can be calculated as in (14). The second moment of service time without considering timeout, can be calculated from (16). However, if dropped packets are included, mean service time of a packet is given by [15]

$$
E\left\{T_{L}\right\}=\left(1-P_{\text {out }}\right) E\left\{T_{L, \text { suc }}\right\}+t_{\text {out }} P_{\text {out }}
$$

and the second moment of the service time including dropped packets is given by

$$
E\left\{T_{L}^{2}\right\}=\left(1-P_{\text {out }}\right) E\left\{T_{L, \text { suc }}^{2}\right\}+t_{\text {out }}^{2} P_{\text {out }}
$$

B. $\min (N, T)$ policy applied to the queuing system

With reference to Fig. 2, let the random variable $K_{i}$, $i=1,2, \ldots$, be the inter-arrival times of packets which are independent, identically, and exponentially distributed with mean arrival rate $\lambda$. Further, let the random variable $A_{n}$ $n=1,2, \ldots$, denote the arrival times, i.e. the epoch when the $n$th packet arrives:

$$
A_{n}=\sum_{i=1}^{n} K_{i}
$$

which has the CDF [4]

$$
F_{n}(t)=\operatorname{Pr}\left\{A_{n} \leq t\right\}=\int_{x=0}^{t} \frac{\lambda(\lambda x)^{n-1}}{(n-1) !} e^{-\lambda t}=\operatorname{Pr}\{N(t) \geq n\}
$$

where $N(t)$ is the number of packets arriving at the system during $[0, t]$. Then, according to $[7$, eq. (6)], the expected number of packets $L_{N T}$ for the $\min (N, T)$ policy and M/G/1 queue is given by

$$
\begin{aligned}
L_{N T} & =L_{0}+\frac{E\{X(X-1)\}}{2 E\{X\}} \\
& =\rho+\frac{\lambda^{2} E\left\{T_{L}^{2}\right\}}{2(1-\rho)}+\frac{\sum_{n=1}^{N}(n-1) F_{n}(T)}{\sum_{n=1}^{N} F_{n}(T)}
\end{aligned}
$$

where $L_{0}$ is the expected number of packets present in an $\mathrm{M} / \mathrm{G} / 1$ queue without $\min (N, T)$ policy, $X$ is a random variable denoting the number of packets waiting in the queue buffer when the busy period begins, and $E\{X\}=\frac{\sum_{n=1}^{N} F_{n}(T)}{F_{1}(T)}$. The parameter $\rho=\lambda E\left\{T_{L}\right\}$ represents the system utilization.

\section{Performance measures for $\min (N, T)$ policy with $M / G / 1$}

In the sequel, several performance metrics are provided that are then utilized to formulate the power consumption function.

1) Expected length of busy period: The expected length of the busy period, $B_{N T}$, can be obtained according to [6] as

$$
E\left\{B_{N T}\right\}=\frac{\sum_{n=1}^{N} F_{n}(T)}{1-e^{-\lambda T}} E\left\{B_{0}\right\}=\frac{E\{X\} E\left\{T_{L}\right\}}{1-\rho}
$$

where $E\left\{B_{0}\right\}$ represents the expected length of the busy period of an $\mathrm{M} / \mathrm{G} / 1$ queue without $\min (N, T)$ policy and $E\left\{B_{0}\right\}=E\left\{T_{L}\right\} /(1-\rho)$.
2) Expected length of idle period: According to [6], the expected length of the idle period, $I_{N T}$, is given by

$$
E\left\{I_{N T}\right\}=\frac{\sum_{n=1}^{N} F_{n}(T)}{1-e^{-\lambda T}} E\left\{I_{0}\right\}=\frac{E\{X\}}{\lambda}
$$

where $E\left\{I_{0}\right\}$ is the expected idle period of an $\mathrm{M} / \mathrm{G} / 1$ queue without $\min (N, T)$ policy. As the durations between two successive arriving packets are independent, identically, and exponentially distributed with mean $1 / \lambda$, we have $E\left\{I_{0}\right\}=1 / \lambda$.

3) Expected length of the busy cycle: The expected length of the busy cycle, $\Omega_{N T}$, can be obtained as [11, eq. (5)]

$$
E\left\{\Omega_{N T}\right\}=E\left\{I_{N T}\right\}+E\left\{B_{N T}\right\}=\frac{E\{X\} E\left\{T_{L}\right\}}{1-\rho}+\frac{E\{X\}}{\lambda}
$$

4) Probability that the radio server is busy: This probability is equivalent to the proportion of time the radio server is busy and therefore given by

$$
P_{B}=\frac{E\left\{B_{N T}\right\}}{E\left\{\Omega_{N T}\right\}}=\frac{E\left\{B_{N T}\right\}}{E\left\{B_{N T}\right\}+E\left\{I_{N T}\right\}}=\lambda E\left\{T_{L}\right\}=\rho
$$

Note that (26) is independent of $N$ and $T$ and therefore the same as for an $\mathrm{M} / \mathrm{G} / 1$ queue without $\min (N, T)$ policy.

\section{Power consumption function}

Let $C_{s}$ denote the setup energy consumption factor. We assume that there is a fixed energy consumption incurred per busy cycle by switching from idle mode to busy mode and vice versa. Further, let $C_{h}$ denote the holding power for each packet present in the system, $C_{b}$ the power consumption while the radio server is in busy state, and $C_{i}$ the power consumption while the radio server is in idle state. Then, the power consumption function, $P_{C}(N, T)$, can be given as [11]

$P_{C}(N, T)=C_{h} L_{N T}+\frac{C_{s}}{E\left\{\Omega_{N T}\right\}}+C_{b} \frac{E\left\{B_{N T}\right\}}{E\left\{\Omega_{N T}\right\}}+C_{i} \frac{E\left\{I_{N T}\right\}}{E\left\{\Omega_{N T}\right\}}$

Then, substituting (22), (23), (24), and (25) into (27) yields

$$
\begin{aligned}
P_{C}(N, T) & =C_{h}\left[\rho+\frac{\lambda^{2} E\left\{T_{L}^{2}\right\}}{2(1-\rho)}+\frac{\sum_{n=1}^{N}(n-1) F_{n}(T)}{\sum_{n=1}^{N} F_{n}(T)}\right] \\
& +C_{s} \frac{\lambda(1-\rho)}{E\{X\}}+C_{b} \rho+C_{i}(1-\rho)
\end{aligned}
$$

\section{NumericAl RESUlts}

In this section, we illustrate the progression of the power consumption function $P_{C}(N, T)$ when keeping $T$ constant and varying $N$ as well as when keeping $N$ constant and varying $T$. The system parameters used in the analysis are provided in the legend of the figures. It is noted that the fading severity parameter is set to $m_{0}=m_{1}=2$ for all links.

Fig. 3 shows that for a given threshold of waiting time $T$ in the queue, the power consumption decreases steeply with the number of packets $N$ increasing from 1 to 6 and then reaches a constant floor. As such, a maximum of $N=6$ packets in the queue appears to be sufficient for reducing the power consumption of the wireless sensor node in this considered scenario. 
Fig. 4 shows the progression of the power consumption function for fixed $N$ and varying $T$. Clearly, if only a maximum of a single packet is queued, i.e. $N=1$, then threshold $T$ has no influence on the power consumption and the largest consumption is observed among the considered scenarios. However, choosing a favorable number of packets $N$ to be accumulated in the queue prior transmission, e.g. $N=6$ or 7 , an increase of the threshold $T$ to around $6 s$ will further decrease the power consumption while larger thresholds of $T$ offer no significant power savings.

As a conjecture of the results shown in Fig. 3 and Fig. 4, the $\min (6,6)$ policy may be applied to this particular scenario in Nakagami- $m$ fading with fading severity parameters set to $m_{0}=m_{1}=2$ for all channels involved.

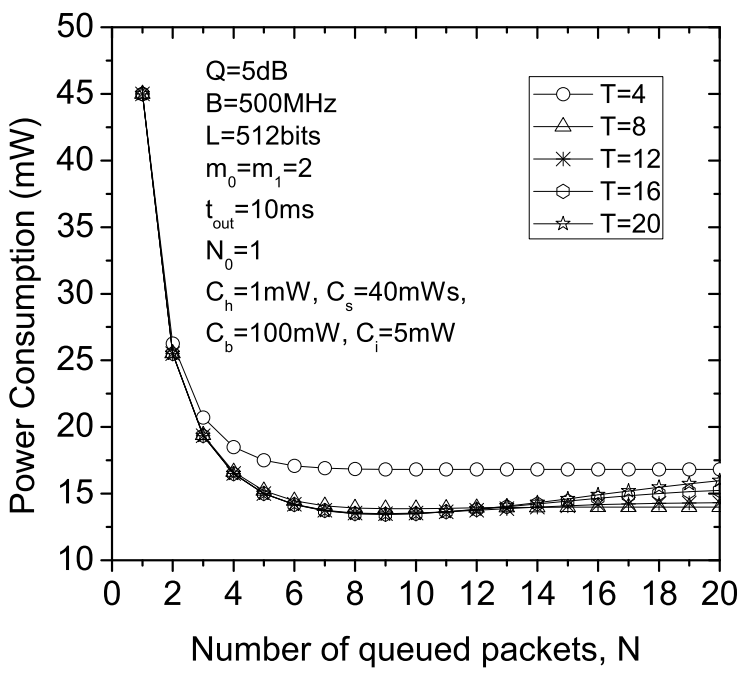

Fig. 3. Power consumption function, $T$ constant.

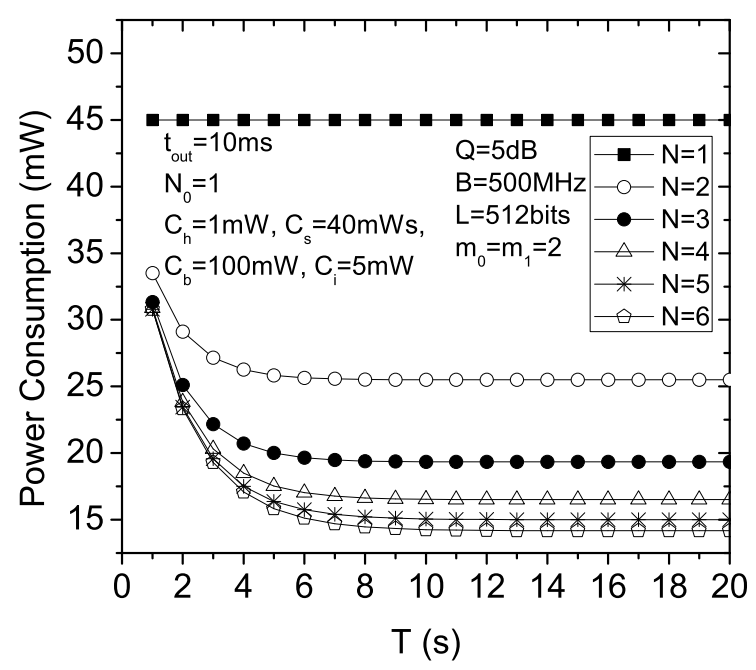

Fig. 4. Power consumption function, $N$ constant.

\section{Conclusions}

In this paper, we have analyzed the power consumption of wireless sensor nodes with $\min (N, T)$ policy and M/G/1 queue. In particular, a queued sleep/wake-up strategy is analyzed in order to mitigate the average power consumption of a wireless sensor node for use in a spectrum sharing environment subject to signal fading. The power consumption function that we obtained depends upon the first two moments of the service time of the data packets, queueing parameters, and inherently accounts for the Nakagami- $m$ fading channel. The numerical examples illustrate how the parameters $N$ and $T$ impact on the power consumption for a given fading scenario.

\section{REFERENCES}

[1] Z. Quan, A. Subramanian, and A. Sayed, "REACA: An efficient protocol architecture for large scale sensor networks," IEEE Trans. Wireless Commun., vol. 6, no. 8, pp. 2924-2933, 2007.

[2] K.-L. Yau, P. Komisarczuk, and P. Teal, "Cognitive radio-based wireless sensor networks: Conceptual design and open issues," in Proc. IEEE 34th Conference on Local Computer Networks, 2009, pp. 955-962.

[3] A. Farraj, S. Miller, and K. Qaraqe, "Queue performance measures for cognitive radios in spectrum sharing systems," in Proc. IEEE GLOBECOM Workshops, Houston, TX, USA, Dec. 2011, pp. 997-1001.

[4] F. C. Jiang, H. W. Wu, D. C. Huang, and C. H. Lin, "Lifetime security improvement in wireless sensor network using queue-based techniques," in Proc. IEEE International Conference on Broadband, Wireless Computing, Communication and Applications, Fukuoka, Japan, Nov. 2010, pp. 469-474.

[5] R. Maheswar and R. Jayaparvathy, "Power control algorithm for wireless sensor networks using N-policy M/M/1 queueing model," Power, vol. 2, no. 07 , pp. $2378-2382,2010$

[6] K. Gakis, H. Rhee, and B. Sivazlian, "Distributions and first moments of the busy and idle periods in controllable M/G/1 queueing models with simple and dyadic policies," Stochastic Analysis and Applications, vol. 13, no. 1, pp. 47-81, 1995.

[7] S. Hur, J. Kim, and C. Kang, "An analysis of the M/G/1 system with $\mathrm{N}$ and T policy," Applied Mathematical Modelling, vol. 27, no. 8, pp. 665-675, 2003.

[8] M. Miller and N. Vaidya, "A MAC protocol to reduce sensor network energy consumption using a wakeup radio," IEEE Trans. Mobile Computing, vol. 4, no. 3, pp. 228-242, May 2005.

[9] V. Casares-giner, P. Wuchner, D. Pacheco-Paramo, and H. De Meer, "Combined contention and TDMA-based communication in wireless sensor networks," in Proc. IEEE EURO-NGI Conference on Next Generation Internet, Karlskrona, Sweden, Jun. 2012, pp. 1-8.

[10] Q. Du and X. Zhang, "Queue-aware spectrum sensing for interferenceconstrained transmissions in cognitive radio networks," in Proc. IEEE International Conference on Communications, Cape Town, South Africa, May 2010, pp. 1-5.

[11] F.-C. Jiang, C.-T. Yang, C.-H. Hsu, K.-H. Wang, and Y.-J. Chiang, "Longevity enhancement technique on IEEE 802.15.4/ZigBee sensor network using D-policy M/G/1 queuing model," in Proc. IEEE International Conference on Computational Science and Engineering, Dalian, China, Aug. 2011, pp. 106-113.

[12] F.-C. Jiang, C.-T. Yang, K.-H. Wang, and D.-C. Huang, "Design framework to optimize power consumption and latency delay for sensor nodes using Min(N, T) policy M/G/1 queuing models," in Proc. IEEE International Conference on Future Information Technology, Busan, South Korea, May 2010, pp. 1-8.

[13] A. Ghasemi and E. S. Sousa, "Fundamental limits of spectrum-sharing in fading environments," IEEE Trans. Wireless Commun., vol. 6, no. 2, pp. 649-658, Feb. 2007.

[14] I. Gradshteyn and I. Ryzhik, Table of Integrals, Series, and Products, 7th ed. Elsevier, 2007.

[15] H. Tran, T. Duong, and H.-J. Zepernick, "Delay performance of cognitive radio networks for point-to-point and point-to-multipoint communications," EURASIP Journal on Wireless Communications and Networking, vol. 2012, no. 1, pp. 1-15, 2012. 